\title{
Finite-Difference Time-Domain Simulation of Low-F\# Fresnel Zone Plates Coupled to IR Antennas
}

\author{
José M. Rico-García, José M. López-Alonso, Brian Lail ${ }^{1}$, Glenn Boreman ${ }^{2}$, Javier Alda \\ School of Optics, University Complutense of Madrid, \\ Avda. Arcos del Jalón s/n, 28037 Madrid, Spain \\ Phone: +34 9139468 72, Fax: +34 913946885 \\ E-mail:jmrico@fis.ucm.es jmlopez@opt.ucm.es, j.alda@fis.ucm.es \\ ${ }^{1}$ Department of Electrical \& Computer Engineering, University of Central Florida \\ P.O Box 162450, Orlando, FL 32816-2450. USA \\ Phone : (407) 823-0676, Fax : (407) 823-5835 \\ Email : blail@mail.ucf.edu \\ ${ }^{2}$ College of Optics and Photonics/CREOL, University of Central Florida. \\ P.O Box 162700, Orlando, FL 32816-2700. USA \\ Phone: (407) 823-6800, Fax: (407) 823-6880 \\ E-mail: boreman@creol.ucf.edu
}

\begin{abstract}
Fresnel Zone Plate Lenses (FZPLs) have been successfully coupled to infrared (IR) antennas producing a responsivity enhancement of about two orders of magnitude. However, their lateral extension may compromise their applicability in focal-planearrays (FPA) IR imagers, where the dimensions of the pixel are constrained by the FPA spacing. When designing optimum-gain FZPLs for FPAs, we are lead to the requirement of FZPLs operating at very low F/\#s (marginal rays propagating at a large angle in image space). In this case, Finite-Difference Time-Domain techniques (FDTD) are used to refine the physical-optics modelling results, producing a result closer to the actual case encountered in a high-fill-factor FPA. In this contribution, we analyze the FZPL designs by using FDTD techniques. The main result of the FDTD computation is the gain factor defined as the ratio of the response of the IR antennas coupled with the FZPL, compared to the same antennas without the FZPL.
\end{abstract}

\section{Introduction}

The use of Fresnel Zone Plate Lenses (FZPL) coupled with IR antennas has been demonstrated recently ${ }^{1}$. The FZPL and the IR antenna were written on opposite sides of a Si wafer. In that study, the focal length of the FZPL was defined by the wafer thickness. After characterizing these devices, it was verified that the sensor responsivity and $D^{*}$ were substantially increased by the use of FZPLs of moderate F\# (ranging from 5.6 to 1.4). Here we have defined the F\# as the ratio between the diameter of the FZPL and its focal length.

The ultimate goal of the FZPL is to improve the specifications of antenna-coupled IR sensors in a focal-plane array (FPA) arrangement. When these IR antennas are located in a twodimensional matrix for imaging applications, the FPA pixel spacing is constrained by crosstalk 
and image-quality considerations. The IR antennas have to be separated a given distance to preclude crosstalk between adjacent detectors. At the same time, the lateral distance between IR antennas defines the pixel size, and therefore, the spatial resolution of the device. When this limitation is applied in the design of FZPL we find that, in order for the FPZL to contain more than one zone, the focal length has to be in the range of the lateral pixel dimension or even smaller. At the same time, these dimensions are very much comparable with the wavelength of the incident radiation. This makes development of an accurate design challenging, since the typical physical-optics approximation where the wavelength is much smaller than the spatial dimensions of the diffracting structures is compromised. Then, a more accurate method involving a simulation tool closer to the actual interaction of light and matter may help to understand the behavior of these devices and corroborate the predicted expectations made with the Fresnel diffraction calculations. The Finite-Difference Time-Domain method (FDTD) is one of the available tools in computational electromagnetics. FZPL have been simulated in the past by other authors using FDTD ${ }^{6,7,8}$. In the case presented in this paper, the calculated parameters given by the FDTD method are driven by the practical goal of improving the design of a FPA pixel containing an IR antenna as the sensor.

Section 2 of this paper is devoted to the dimensional specification of proposed FZPL coupled to IR antennas. Some alternatives are presented and discussed before performing the FDTD simulations. A detailed analysis of the FDTD simulation condition applicable to the FZPL is made in section 3. The results obtained by the FDTD are presented and discussed in section 4. A comparison with the expectations obtained by the Fresnel diffraction calculation is also performed. Finally, section 5 summarizes the main conclusions of the paper.

\section{FZPL design for FPA imaging devices using IR antennas}

Several FZPL designs can be proposed for coupling the radiation to the IR antenna. In this analysis we have assumed that the antenna is located on the surface of a Si wafer coated with an insulting $\mathrm{SiO}_{2}$ layer. In all the designs the focal length is smaller than the pixel size. This corresponds with a low F\# (less than 1). The light is assumed to be incident from the air side. We have proposed three different configurations, assuming that the focal distance is given by the separation between the center of the FZPL and the center of the antenna. Figure 1a represents a typical plane FZPL suspended on the wafer and separated by a distance equal to the focal length. The number of zones within a given pixel size decreases as the focal length increases. This is because the $\lambda / 2$ optical-path difference between the consecutive zones needs more transversal space (larger pixel) as the focal length grows. Following the conclusions derived from a previous paper $^{1}$ we propose to operate the FZPL in a transmissive mode. In Figure $1 \mathrm{~b}$ we present another alternative working in a reflective mode. In order to compensate for the losses produced by the obscuration of the FZPL by the antenna structure and the connection lines, we have designed a reflective FZPL where the successive Fresnel zones are located closer to the antenna. This configuration is also limited by the pixel-size constraint.

Figure 1 shows the basic form of the FZPL under analysis. The FZPL is defined by plane Air/Si interfaces. The size and depth of the interfaces are given by computing the optical path of a plane wavefront coming from the air and focusing on the center of the antenna location. In making such analysis we have assumed that the zone boundaries of the FZPL appear at the locations where the differences in optical path is equal to $\lambda / 2$, with $\lambda=\lambda_{0} / n_{\mathrm{Si}}$. In our case $\lambda_{0}$ is the wavelength in vacuum, which we take as $10.6 \mu \mathrm{m}$, and $n_{\mathrm{Si}}$ is the index of refraction of the 
material used to fabricate the FZPL on the wafer that is assumed to be $\mathrm{Si}, n_{\mathrm{Si}}=3.42$. By applying these two conditions we can define the following two surfaces:

$$
\begin{aligned}
& z_{f^{\prime}}=\frac{f^{\prime} n_{S i}}{n_{S i}+1}\left(1-\sqrt{1-\frac{x^{2}}{f^{\prime 2}} \frac{n_{S i}+1}{n_{S i}-1}}\right), \\
& z_{f^{\prime \prime}+\lambda / 2}^{\prime}=f^{\prime}-\frac{n_{S i}}{\lambda_{o}} \sqrt{\left[x^{\prime 2}-x^{2}-\left(\frac{f^{\prime}}{2 n_{S i}}\right)^{2}\right]^{2}-\frac{\lambda_{o}^{2} x^{2}}{n_{S i}^{2}}},
\end{aligned}
$$

where $f^{\prime}$ is the focal length of the lens, $x$ and $y$ represents the location of the points having constant optical path from the input plane of the FZPL to the center of the antenna. The optical axis is perpendicular to the wafer surface and crosses through the center of the antenna. The input plane of the FZPL is defined as the plane parallel to the wafer surface and separated a distance $f^{\prime}$ from the plane where the antenna is located. Equation (2) represents the relation between the coordinates $x^{\prime}, z^{\prime}$ of a surface having an extra optical path of $\lambda / 2$ with respect to the optical path defined by equation (1) at a constant value of $z$. Therefore, by using equations (1) and (2) it is possible to define the zone boundaries of the FZPL. The origin of the coordinate system, $O$, for $(x, y)$ and $\left(x^{\prime}, y^{\prime}\right)$ is placed at the intersection of the optical axis and the first zone.

Equation (1) deserves special attention because it is possible to derive a condition about the minimum $F \#$ obtainable from the proposed design. In order to have a real number as the solution for $y$, at the maximum value of $x=R$ (being $R$ the half size of the pixel), $F \#$ has to be larger than,

$$
F \#_{\text {min }}=\frac{f^{\prime}}{2 R}=\frac{1}{2} \sqrt{\frac{n_{S i}-1}{n_{S i}+1}} .
$$

In our case this lower bound is $F \#_{\min }=0.68$.
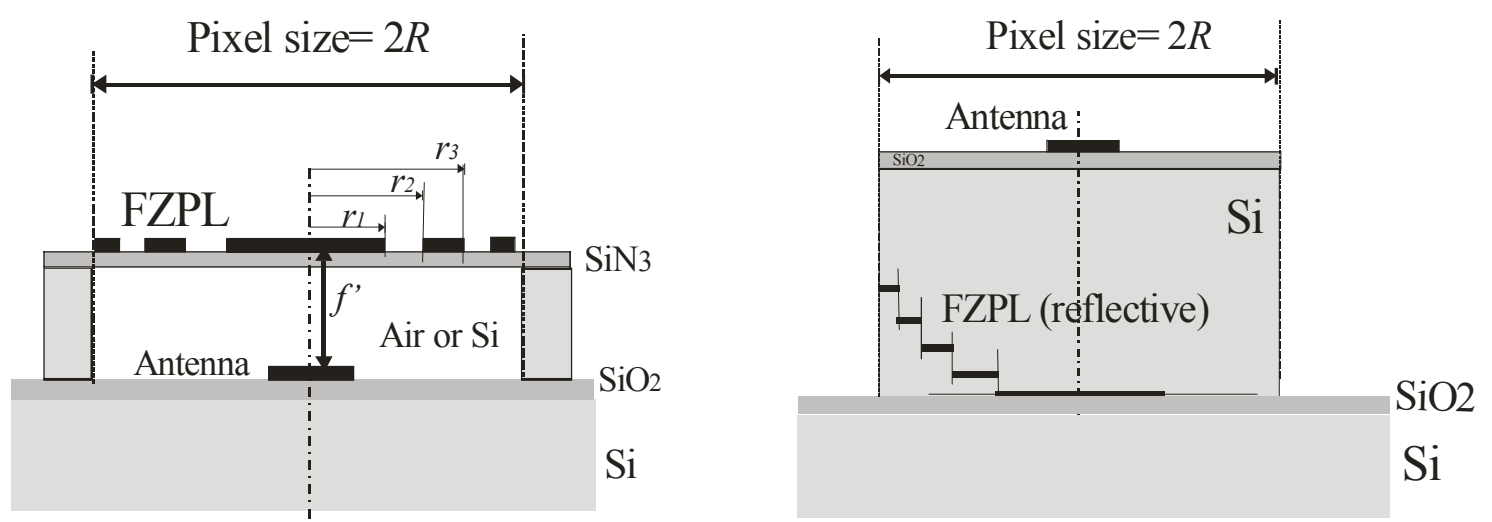

Figure 1: Configuration of two designs of FZPL.

a) Left: FZPL suspended on a plane layer and separated a distance equal to the focal length.

b) Right: Kinoform reflective FZPL.

It is also possible to consider the construction of phase plates by dimensioning the profile of the FZPL with $\lambda / 2$ indentations. This third configuration is represented in Figure 2, corresponding to a FZPL having a staircase geometry, known as a kinoform ${ }^{2}$. The practical calculation of a given FZPL having a kinoform shape and working in transmissive mode uses equations (2) and (3) as follows. First, the focal length, $f^{\prime}$, the index of refraction of the material filling the space 
between the FZPL and the antenna, $n$, and the wavelength, $\lambda$, are known. The origin of coordinates is located at the pole of the lens surface, and at a distance $\mathrm{f}^{\prime}$ from the antenna center. The first Fresnel zone extends from the point $\left(x_{1}, 0\right)$, to a distance given by equation (3) after substituting $x=z=0$. The following zone begins at a different height from the wafer. This height is calculated from equation (2) for $x=x^{\prime}{ }_{1}$. Then, the coordinates of the second step is given by $\left(x_{2}, z_{2}\right)$. We have assumed that the first step is characterized by $\left(x_{1}=0, z_{1}=0\right)$. Now, we can apply equation (3) successively to obtain the consecutive Fresnel zones until reaching the maximum available value of $x=R$, being $R$ half the size of the pixel.

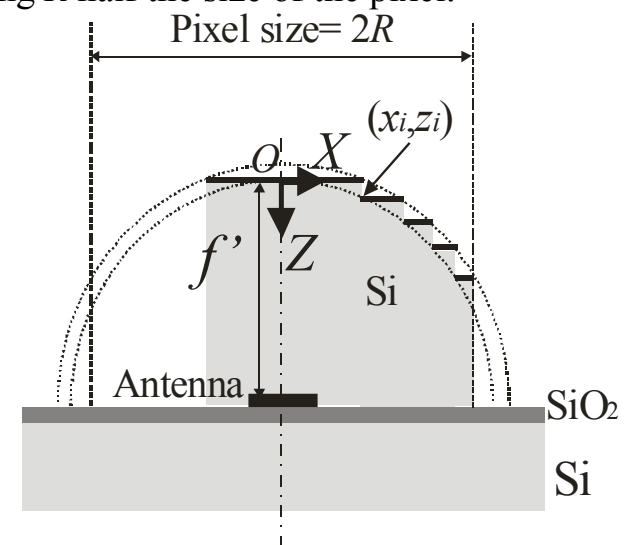

Figure 2: Graphical representation of the geometry of the kinoform transmissive FZPL. The Fresnel zones are characterized by the coordinates of the points $\left(x_{i}, y_{i}\right)$ where $i$ runs from 1 to the maximum available number of zones allowed within the pixel size.

2.1.- Dimensioning and diffractional performance of a FZPL kinoform working in transmissive form

In this subsection we present the results of the previously described method applied to the design of a FZPL having an axial symmetry. The illumination wavelength is $\lambda_{0}=10.6 \mu \mathrm{m}$, and the material filling the kinoform is $\mathrm{Si}, n_{\mathrm{Si}}=3.42$. The focal length is equal to $f^{\prime}=20 \mu \mathrm{m}$ and the pixel size is $2 R=29.70 \mu \mathrm{m}$. The coordinates of the steps are presented in Table 1 .

\begin{tabular}{|c|c|c|}
\hline$i$ & $x_{i}$ (microns) & $z_{i}$ (microns) \\
\hline 1 & 0 & 0 \\
\hline 2 & 8.02 & 2.47 \\
\hline 3 & 11.25 & 5.42 \\
\hline 4 & 13.64 & 9.47 \\
\hline
\end{tabular}

Table 1: Dimensions of FZPL

The $F \#$ of this FZPL is equal to the minimum value of 0.68 . This kinoform FZPL contains four Fresnel zones, but just three of them are complete.

The performance of this FZPL can be described by a gain factor. This gain factor is defined as the ratio between the irradiance at the center of the antenna when the FZPL is placed above it, with respect to the irradiance obtained without the FZPL. In this case the gain factor reaches a value of 9.53. Figure 3 shows the evolution of the gain factor as the focal length increases. It should be noted that the designs analyzed here have a minimum value of the $F \#$. Therefore, if we limit the size of the pixel to $50 \mu \mathrm{m}$, then the focal length has also a limitation at $34 \mu \mathrm{m}$. 


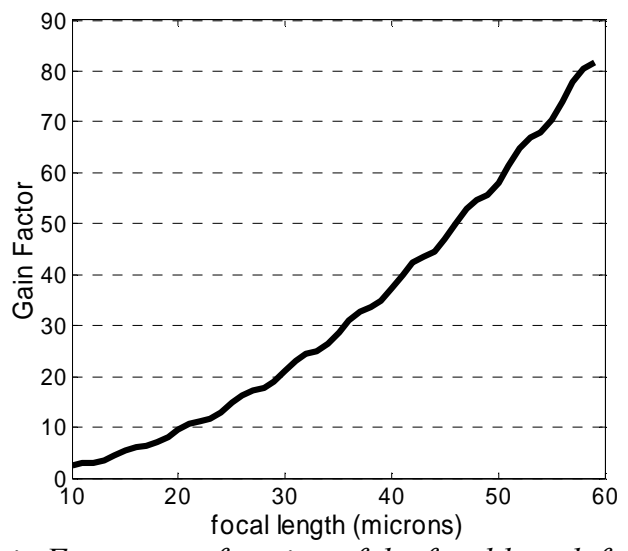

Figure 3. Evolution of the Gain Factor as a function of the focal length for transmissive kinoforms FZPL having a minimum $F \#=0.68$.

The diffractive calculation allows the evaluation of the irradiance map at any location behind the FZPL (see Fig. 4). It should be noted that this calculation is made in the scalar approximation of the electric field. Therefore, it is not able to describe any polarization dependence. Then, the results obtained from this calculation should be taken only as an approximation. The FDTD simulation that actually models the interaction of the electromagnetic fields, and their evolution along the space and time, should provide a more faithful representation of the actual situation.

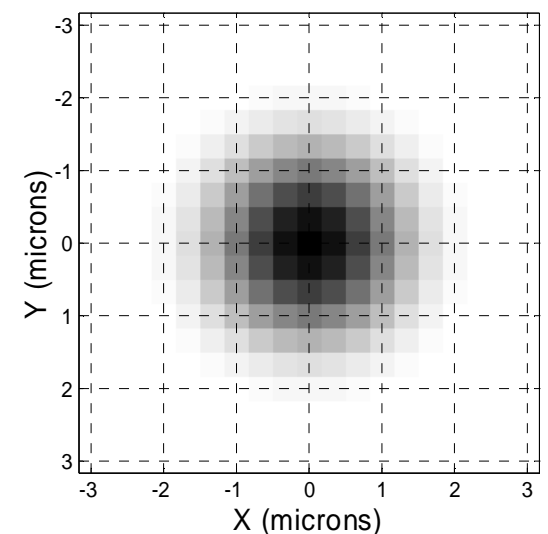

Figure 4: Map of the irradiance at the position of the focus for a kinoform FZPL working in transmissive mode and having a focal length of $20 \mathrm{~mm}$.

\section{FDTD simulation of proposed structures}

This section is devoted to the simulation of the transmissive kinoform introduced above by making use of a well-established tool in computational electromagnetics: the Finite Difference Time-Domain method ${ }^{3}$. Its application in the IR and optical range has increased during the last ten years, giving rise to accurate predictions and useful designs for a great variety of photonic devices ${ }^{4,5}$. The use of FDTD to compute focusing properties of diffractive structures is not new $^{6,7,8}$, even in the IR part of the spectrum?; however, its systematic application is not as common as other computational methods. 
3.1.- Main equations and parameters of the simulations.

In order to develop a model which recovers the basic issues of the interaction between the IR radiation and the structure under study, we have chosen a two-dimensional approach (2D). In two dimensions, Maxwell equations decouple in two sets with different polarization properties: Transverse Electric (TE), and Transverse Magnetic (TM). This last set is composed of three non-trivial components of the electromagnetic field, namely, $E_{z}, H_{x}$, and $H_{y}$ - if the $z$ axis is taken as the axis orthogonal to the plane where the $\mathrm{H}$ fields components lie. Then, the Maxwell equations for the TM set read as

$$
\begin{aligned}
& \partial_{t} H_{x}=\frac{1}{\mu}\left(-\partial_{y} E_{z}\right), \\
& \partial_{t} H_{y}=\frac{1}{\mu}\left(\partial_{x} E_{z}\right), \\
& \partial_{t} E_{z}=\frac{1}{\varepsilon}\left(\partial_{x} H_{y}-\partial_{y} H_{x}\right) .
\end{aligned}
$$

These equations are solved following the usual procedure outlined by Yee and others ${ }^{10}$. Some relevant parameters of the simulation are listed in Table 2.

\begin{tabular}{|c|c|c|c|c|c|c|c|}
\hline $\mathrm{S}$ & $\Delta(\mu \mathrm{m})$ & $\Delta \mathrm{t}(\mathrm{s})$ & Excitation & $\mathrm{N}_{\lambda \min }$ & $\begin{array}{c}\text { Simulation } \\
\text { Time }\end{array}$ & $\mathrm{ABC}$ & $\begin{array}{c}\text { Grid Size } \\
\mu \mathrm{m} \times \mu \mathrm{m}\end{array}$ \\
\hline 0.7 & 0.1033 & $2.412 \times 10^{-16}$ & $\mathrm{TF} / \mathrm{SF}$ & 30 & $8000 \Delta \mathrm{t}$ & $\mathrm{UPML}$ & $56.61 \times 29.60$ \\
\hline
\end{tabular}

Table 2: $\boldsymbol{S}$ is the Courant Factor; $\boldsymbol{\Delta}$ is the spatial step; $\boldsymbol{\Delta}$ t is the temporal step; $\boldsymbol{N}_{\lambda m i n}$ is the points per minimum wavelength in the grid; the absorbing boundary condition (ABC) is an Uniaxial Perfect Matched Layer (UPML) of 14 cells.

\section{2.- Absorbing boundary conditions and excitation of the grid.}

As pointed out in Table 1, the absorbing boundary condition employed in this work is the Uniaxial Perfect Matched Layer (UPML). Specifically, it is designed to achieve a reflection coefficient under normal incidence around $1 \times 10^{-10}$ and it is 14 cells thick. We have observed no spurious reflections greater than $1 \times 10^{-7}$ in large oblique angles during all the simulation time, thus allowing a reliable computation of the fields inside the grid. To excite the lens, we have launched into the grid a plane wave in normal incidence through a Total Field-Scattered Field (TF/SF) splitting of the physical domain ${ }^{3}$, having a very narrow spectral content peaked at $\lambda_{0}=$ $10.6 \mu \mathrm{m}$. Explicitly, the time signature of the excitation is:

$$
f(t)= \begin{cases}e^{-\left(\frac{t-t_{0}}{T}\right)^{2}} \sin \left(2 \pi v_{0} t\right) & \mathrm{t}<\mathrm{t}_{0} \\ \sin \left(2 \pi v_{0} t\right) & \mathrm{t} \geq \mathrm{t}_{0}\end{cases}
$$

Where $v_{0}$ is $28.282 \mathrm{THz}$, and $t_{0}$ and $T$ are fixed carefully to get a smooth transition from the raising tail of the pulse to the monochromatic oscillation train. A graphical representation of the time evolution of the excitation source is plotted in Fig. 5. 


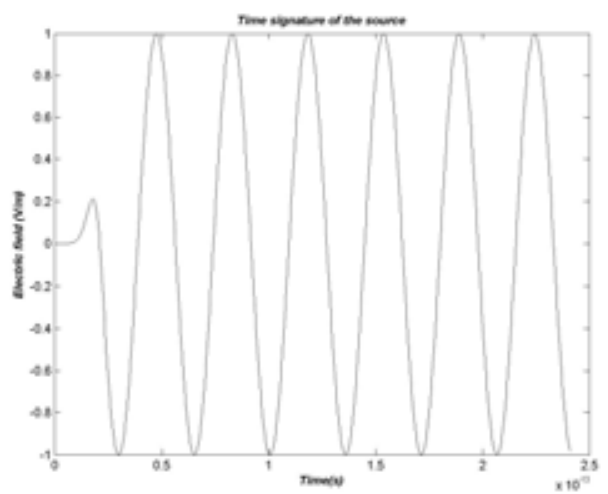

Figure5: Time signature of the source employed to excite the grid

\section{3.- Features of the computed fields.}

To study the lens properties, we have carried out simulations where the electric field has been recorded in the whole grid each 250 time steps. Figure 6 displays a snapshot of the square absolute value of the electric field. As can be seen, the lens is focusing in the zone expected from the scalar case, except for small deviations explained for the vector nature of the electromagnetic field ${ }^{9}$.

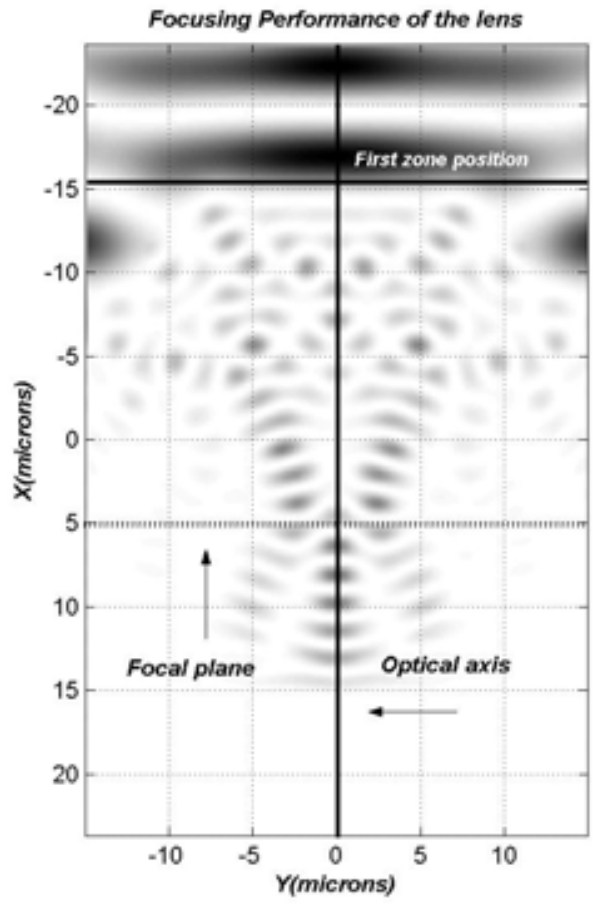

Figure 6: Focusing performance of the lens.

It should be noticed the great amount of power reflected back by the structure. This issue will be discussed in Section 4. Figure 7 shows the temporal focusing sequence of the plane wave impinging in normal incidence over the lens. 

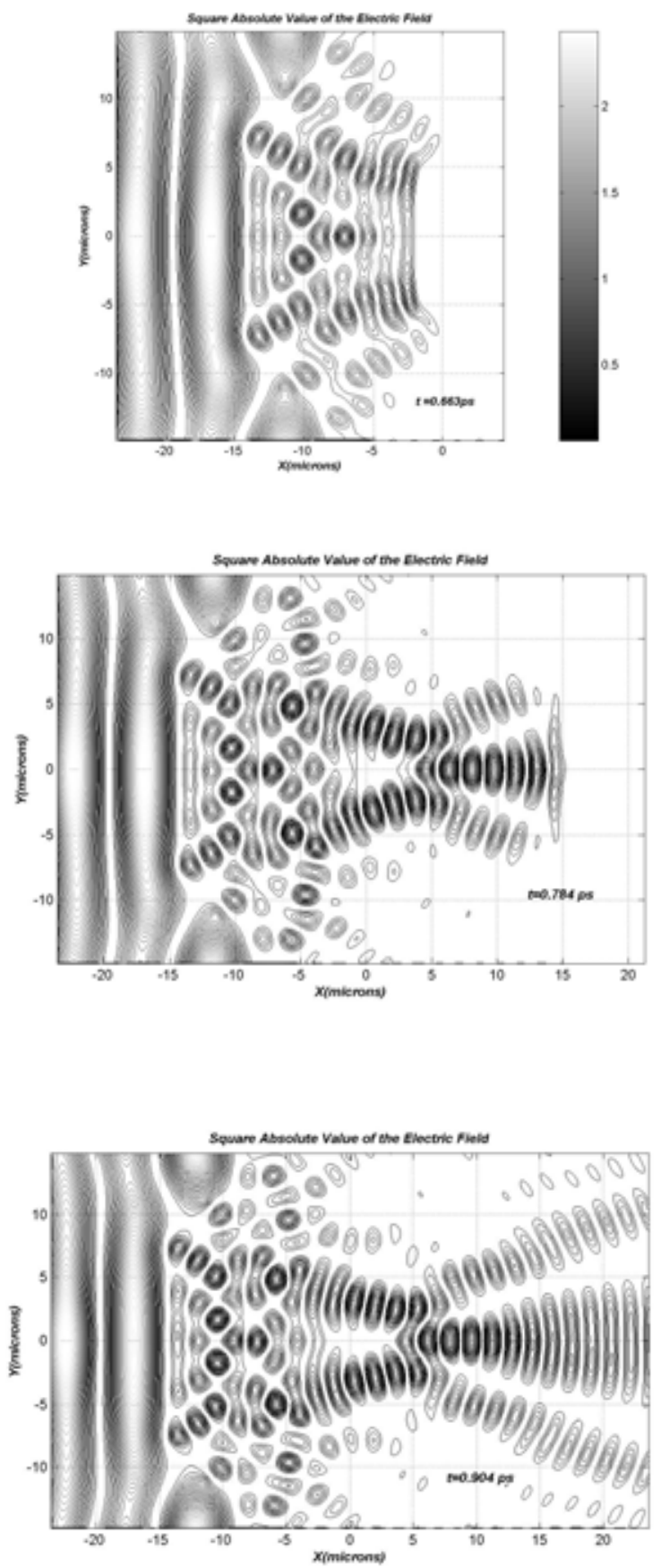

Figure 7. Top: Snapshot of the square modulus of the electric field. The plane wave incoming from left is being focused by the lens, although a significant amount of power is reflected back by the structure. Center: Snapshot of the square absolute value of the electric field at $t=0.784$ ps. Bottom: Snapshot of the square absolute value of the electric field at $t=0.904 \mathrm{ps}$. Now, the ray cone has been formed, and there is a well-defined focal region where the optical power is being concentrated 


\section{Analysis and Discussion}

\subsection{Lens parameters from FDTD simulations.}

To have a deeper insight on the focusing mechanism of the kinoform and to quantify its properties, we have recorded the fields in the focal region once the unavoidable transients introduced by the impulsive source have disappeared from the grid. We have performed a Fast Fourier Transform (FFT) on the whole focal region, after recording the E field during the last 700 time steps of the simulation. Then we have picked up the Fourier component at the excitation frequency $(\lambda=10.6 \mu \mathrm{m})$ in every point in the focal region. To have a measure of the optical power, and to compare with the scalar case, we use the square absolute value of the Fourier component at the excitation frequency, $\omega_{0}$ :

$$
I\left(\omega_{0}\right) \cong\left|E z\left(x, y ; \omega_{0}\right)\right|^{2}
$$

The derivation of lens parameters is straightforward after computing the irradiance, except for the gain factor. The main parameters are listed in Table 3.

\begin{tabular}{|c|c|c|}
\hline Focal distance, $f^{\prime}(\mu \mathrm{m})$ & Focal shift, $\Delta f^{\prime}(\mu \mathrm{m})$ & Depth of focus $(\mu \mathrm{m})$ \\
\hline 20.66 & +0.66 & 0.9297 \\
\hline
\end{tabular}

Table 3: Parameters of the lens obtained from the FDTD simulation

Where the focal shift is defined as the difference between the expected value computed using the scalar approach of the focal distance and the actual value computed with the FDTD algorithm. The depth of focus is defined as the size of the region where the intensity is greater than $80 \%$ of the intensity at the focus 9 .

\subsection{Gain factor.}

To compute the gain factor requires an extra simulation using the same parameters but excluding the lens from the grid. Then, the same procedure applied previously is used to find the measure of the irradiance without the lens in the focal plane. The quotient of both quantities is defined as the Gain Factor. A computation of it is shown in figure 8.

As can be noted from figure 8, the scalar approach result obtained in Section 2 is similar to the vectorial one, providing both similar values for the gain. Slight differences in the Gain Factor and the focal length can be attributed to the interaction of radiation with subwavelength features of the lens, not suitably recovered by the scalar formulation. Besides, the gain factor computed with the physical-optics approximation is calculated on the antenna plane, whereas the FDTDcomputed gain provide information along the optical axis. Moreover, some differences should be expected if there is a change in the polarization of the incidence plane wave ${ }^{9}$. 


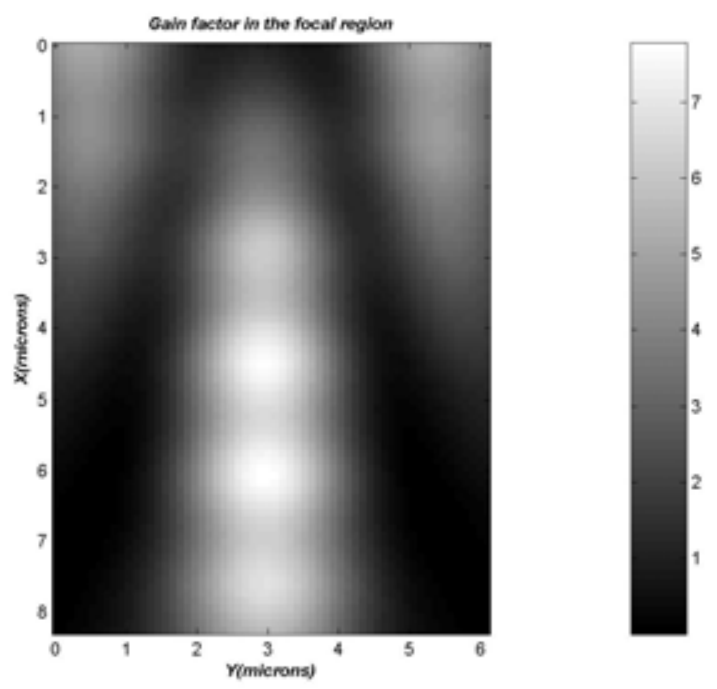

Figure 8: Gain Factor in the focal region

\section{Conclusions}

In this contribution we have proposed several designs of FZPLs for its use with IR antennas. One of the designs, the kinoform FZPLs working in transmissive mode, has been analyzed first by using the scalar diffraction approach. The low $F \#$ of the proposed design has suggested the evaluation of the FZPL behaviour by using FDTD algorithms. The results obtained from the FDTD are compared with the scalar diffraction results. FDTD has been able to predict a focal shift. On the other hand, the gain factor obtained from the two models are comparable.

Summarizing, FDTD two-dimensional simulations provide invaluable information about the actual behaviour of the electric field impinging on the IR antenna. When including the dispersive properties of the metal structure into the algorithm, it will be possible to evaluate the currents excited in the antenna, and therefore the expected signal obtained from the detector. Further refinements of the technique can be included to take into account the three dimensional character of the structure.

\section{Acknowledgments}

This work has been partially supported by the Ministerio de Ciencia y Tecnología of Spain through the project TIC2001-1259, and by the Collaboration Agreement between the University Complutense of Madrid (Spain) and the University of Central Florida (FL, USA).

\section{References}

1.- F. J. González, J. Alda, B. Ilic, G. D. Boreman, IR Antennas coupled to Lithographic Fresnel Zone Plates. Appl. Opt., 43, (in press) (2004).

2.- H. D. Hristov, "Fresnel zones in wireless links, zone plate lenses and antennas",Artech House, Norwood MA, 2000. 
3.- Taflove and S. C. Hagness Computacional Electrodynamics: The Finite-Difference Time Domain Method, $2^{\text {nd }}$ ed. (Artech House, Boston, 2000).

4.- J.B.Judkins, R.W.Ziolklowski, “ Finite-Difference Time-Domain Modeling of Nonperfectly Conducting Metallic Thin-Film Gratings", J. Opt. Soc. Am. A, 12, 1974-1983, (1995).

5.- O. Painter, R. K. Lee, and A. Scherer, "Two-dimensional photonic band-gap defect mode laser," Science, 284, 1819-1821, (1999).

6.- W.B.Dou, "Analysis of frequency dependence and focusing performance of diffractive lens", Opt. Express, 10, 1018-1027, (2002)

7.- D.W. Prather, S. Shi, "Formulation and application of the finite-difference time-domain method for the analysis of axially symmetric diffractive optical elements," J. Opt. Soc. Am. A, 16, 1131-1142 (1999).

8.- Mark S. Mirotznik, Dennis W. Prather, Joseph N. Mait, William A. Beck, Shouyuan Shi,and Xiang Gao, "Three-dimensional analysis of subwavelength diffractive optical elements with the finite-difference time-domain method", Appl. Opt., 39 (17), 2871-2880, (2000)

9.- D. Feng, Y. Yan, G. Jin, S. Fan, "Beam focusing characteristics of diffractive lenses with binary subwavelength structures", Opt. Commun. (in press) (2004).

10.- K. S. Yee, "Numerical solution to initial boundary value problems involving Maxwell's equations in isotropic media," IEEE Trans. Antennas Prop., AP14, 302, (1966). 